\section{3-D Stereoscopic Display of High Resolution Flat Panel Computed Tomography Temporal Bone Imaging}

\section{Matthew Kiczek $^{1 *}$, Meiyappan Solaiyappan², Monica Pearl' and James Zinreich ${ }^{1}$}

${ }^{1}$ Neuroradiology Division, The Russell H. Morgan Department of Radiology and Radiological Science, The Johns Hopkins Hospital, Baltimore, USA

${ }^{2}$ Radiology Research Division, The Russell H. Morgan Department of Radiology and Radiological Science, Baltimore, USA

\begin{abstract}
\section{Study background}

The objective of this preliminary presentation is to show the advantages of the application of 3D stereoscopic imaging technology to high resolution flat panel computed tomography in the evaluation of normal and pathologic temporal bone.
\end{abstract}

\section{Methods}

Our study represents a retrospective evaluation of the temporal bones of five patients with high resolution flat panel computed tomography imaging data performed on Dyna CT (Siemens) between July 2017 and July 2018. Three of these patients were considered to be normal, one had a stapes prosthesis and one had and superior semicircular canal dehiscence. The data from these imaging examinations was de-identified and uploaded into a 3-D stereotactic imaging display unit (an advanced evolution of the Dextroscope imaging device), and virtually evaluated and manipulated in a three-dimensional virtual environment by board certified neuroradiologists.

\section{Results}

The flat panel CT imaging data viewed and evaluated on the 3-D stereotactic imaging display unit provided an intuitive, clear, three dimensional and highly detailed evaluation of the temporal bone anatomy. Details not well evaluated on conventional temporal bone

*Corresponding Author: Matthew Kiczek, Neuroradiology Division, The Russell H. Morgan Department of Radiology and Radiological Science, The Johns Hop kins Hospital, Baltimore, USA, Tel: +1 4408648074; E-mail: mkiczek1@jhmi.edu

Citation: Kiczek M, Solaiyappan M, Pearl M, Zinreich J (2019) 3-D Stereoscopic Display of High Resolution Flat Panel Computed Tomography Tempora Bone Imaging. J Otolaryng Head Neck Surg 5: 36

Received: August 15, 2019; Accepted: September 09, 2019; Published: September 16, 2019

Copyright: (c) 2019 Matthew Kiczek, et al. This is an open-access article distributed under the terms of the Creative Commons Attribution License, which permits unrestricted use, distribution, and reproduction in any medium, provided the original author and source are credited.
$\mathrm{CT}$, such as on the cases with pathology, were brought into clearer light. Furthermore, the ability to manipulate the imaging data in a 3D workspace provided an additional dimension and clarity to the regional anatomy and specifically the ossicular chain.

\section{Conclusion}

Evaluation of this high resolution data on a 3-D stereotactic imaging display would serve to benefit several regional morphologic evaluations. The ability to manipulate the images in a 3-D workspace could greatly benefit surgeons in their pre-operative planning and post-operative evaluations. Regarding the temporal bone, precise evaluation of ossicular pathology, cochlear implant position and function would be facilitated. This presentation provides a preliminary display of the above mentioned capabilities.

Keywords: Flat-Panel CT; Temporal bones; Three-dimensional

\section{Introduction}

There is much utility in the detailed analysis of the intricate temporal bone anatomy and understanding the morphologic three dimensional relationships. Understanding the anatomic architecture of the temporal bone is of great utility for otolaryngologists, both in the pre and post-operative state. Conventional high resolution evaluation of the temporal bone provides adequate anatomic evaluation but ultrahigh resolution temporal bone imaging and evaluation with flat panel detector computed tomography has shed light on anatomic intricacies and pathologic processes not well delineated on the conventional imaging evaluation. Subtle pathologic processes, particularly those involving the small and delicate ossicular structures, are seen to greater advantage on the ultrahigh resolution imaging acquisitions.

Evaluation of the temporal bone structures on a conventional workstation is the standard. Utilizing the ultrahigh resolution imaging data to view the detailed anatomic relationships in a virtual three-dimensional environment has not before been performed. Utilizing three-dimensional stereoscopic imaging with an evolution of the Dextroscope imaging device, we were able to add a new dimension to evaluating normal and pathologic temporal bones.

The objective of this preliminary study is to show the advantages of applying 3D Stereoscopic Imaging (3DSI) technology to high resolution Flat Panel Computed Tomography (FPCT), in the evaluation of normal and pathologic temporal bone. Evaluation of high resolution FPCT source data augmented with 3DSI display has not been reported to date. Our objective is to show the advantage of combining these imaging technologies and show the potential benefit in the evaluation of the temporal bone anatomy and pathology.

\section{Methods}

Our study represents a retrospective evaluation of the temporal bones of five patients with high resolution FPCT imaging data performed on Dyna CT (Siemens) between July 2017 and July 2018. Four of these patients were considered to be normal, one had stapes prosthesis and one had superior semicircular canal dehiscence. 
Citation: Kiczek M, Solaiyappan M, Pearl M, Zinreich J (2019) 3-D Stereoscopic Display of High Resolution Flat Panel Computed Tomography Temporal Bone Imaging. J Otolaryng Head Neck Surg 5: 36

The high resolution data from these imaging examinations was de-identified and uploaded into a 3DSI display unit (an advanced evolution of the Dextroscope imaging device), and virtually evaluated by board certified neuroradiologists.

An essential key feature of our software is that it provides a $3 \mathrm{D}$ stereoscopic representation of the anatomy that can be viewed using a pair of electronic glasses, which helps us perceive the depth of an object, just as one would perceive it, naturally, in the "real-world". To date, our challenge is to create the same effect, as we display the $3 \mathrm{D}$ formatted structure, created by our software, onto a "flat" paper media, without having the viewer use of electronic glasses, in a reasonably practical or economic way. Therefore, the results are presented as volume-rendered images displayed on a flat surface, without the depth perception effect, and therefore a "simulated" 3D display, or if we might be allowed to say "a $2.5 \mathrm{D}$ display".

\section{Results}

A total of five patients who received imaging using FPCT were evaluated. Imaging evaluation was performed by board certified neuroradiologists in a preliminary evaluation of the three-dimensional imaging software. The temporal bone data was able to be manipulated in a 3D workspace utilizing handheld controllers while wearing special electronic glasses. Portions of the temporal bone could be removed with "virtual surgery" to obtain a clearer view of specific anatomic structures or pathologic processes. In this process, portions of the normal anatomy were virtually manipulated and removed in order to better appreciate certain regions.

Several obliquities of the Superior Semicircular Canal Dehiscence (SSCD) were photographed in a " $2.5 \mathrm{D}$ " projection to add depth. The site of dehiscent arcuate eminence was clearly seen (Figure 1). In addition, a patient with a history of stapes prosthesis placement was evaluated. Again, using virtual surgery and 3D manipulation, various obliquities of the prosthesis were produced (Figure 2).

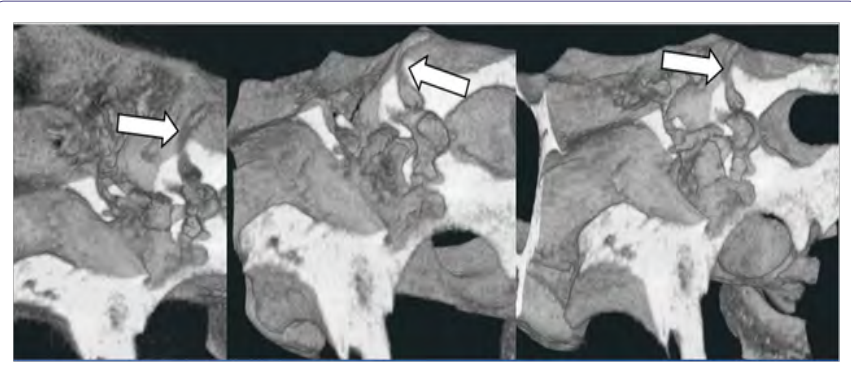

Figure 1: Oblique cutaways into the 3-D volume rendered display demonstrating a dehiscent superior semicircular canal in various obliquities (large white arrows).

Evaluation of the normal temporal bone studies was also undertaken. 3D manipulation and virtual surgery produced detailed images showing the relationship of the ossicular chain in different obliquities. These projections cannot be produced with conventional temporal bone imaging. The normal relationship of the malleolus, incus, and stapes in three dimensions was also clearly elucidated (Figures 3-5).

\section{Discussion}

Microstructures the FPCT imaging data viewed and evaluated on the 3DSI unit provided an intuitive three-dimensional detailed evaluation of the temporal bone anatomy. Details not well evaluated on conventional temporal bone CT were seen to greater advantage. Furthermore, the ability to manipulate the imaging data in a 3D workspace and perform "virtual surgery" provided an additional dimension and clarity to the sophisticated regional temporal bone micro structures.

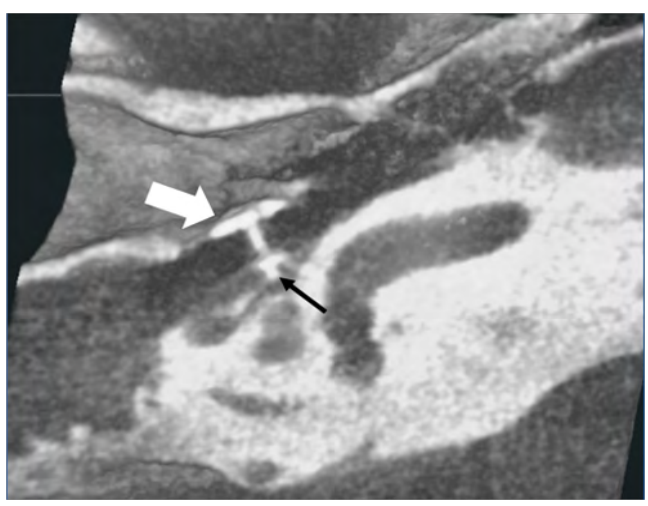

Figure 2: Oblique cutaway into the 3-D volume rendered display demonstrating stapes prosthesis (large white arrow) and its articulation with the oval window (small black arrow).

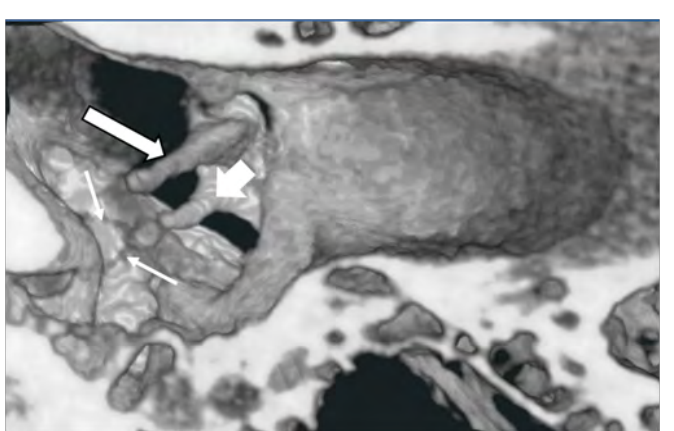

Figure 3: Oblique cutaway into the 3-D volume rendered display demonstrating the relationship of the malleus (white arrow), incus (white arrowhead), and the stapes (small white arrows).

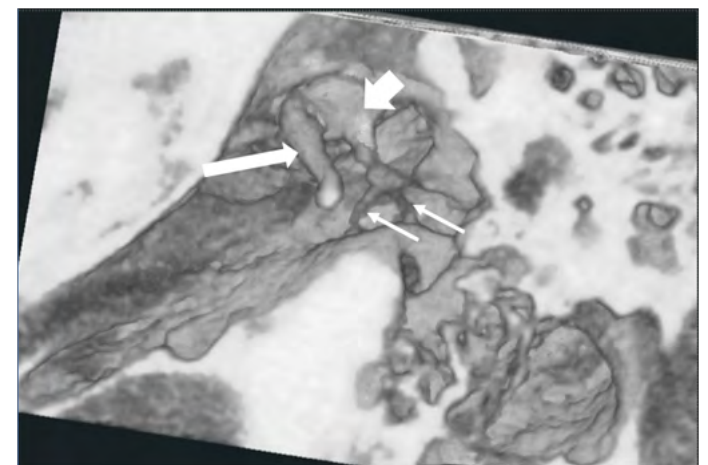

Figure 4: Oblique cutaway into the 3-D volume rendered display again demonstrating the relationship of the malleus (white arrow), incus (white arrowhead), and the stapes (small white arrows). 


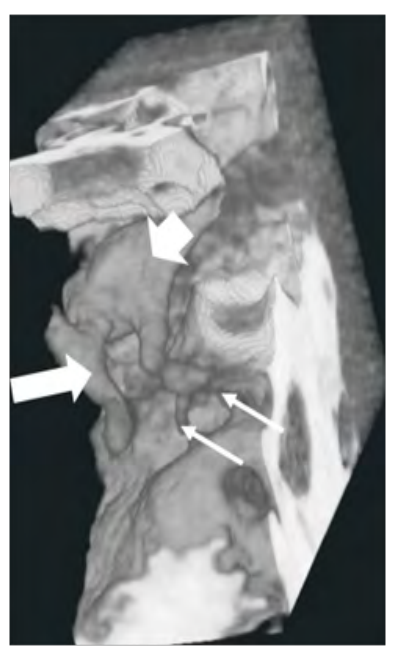

Figure 5: Oblique cutaway status post virtual surgery into 3D volume of data to more optimally display the ossicular anatomy. The relationship of the malleus (white arrow), incus (white arrowhead), and the stapes (small white arrows) are again shown.

The intricate anatomic architecture of the temporal bone has been investigated extensively. Most recently, Ultra-High Resolution Computed Tomography (UHRCT) techniques using $0.5 \mathrm{~mm}$ to $0.25 \mathrm{~mm}$ collimation have been used to image the temporal bone structures in greater detail. An investigation by Yamashita, et al., demonstrated the anatomy of the temporal bone to be more conspicuous on UHRCT than on conventional CT [1]. Evaluation of the temporal bone infrastructure has also been performed with FPCT with voxel sizes ranging from 0.08 to $0.1 \mathrm{~mm}$. In FPCT imaging, scanning is performed with a volumetric $\mathrm{x}$-ray tube with a wider anode angle [2]. A flat panel detector is used, consisting of a film of cesium iodide scintillator crystals [2]. After the imaging data undergoes a reconstruction algorithm, images with significantly higher spatial resolution and slightly higher contrast resolution than conventional multidector $\mathrm{CT}$ are produced [2].

Bartling, et al., looked at high resolution FPCT in preoperative temporal bone patients [3]. Evaluation at such high resolution produces a clear and detailed picture of both normal and pathologic temporal bones. Studies using the high resolution technique have been performed comparing it to conventional $\mathrm{CT}$ imaging techniques in both normal and pathologic conditions [4]. The conspicuity of the temporal bone anatomic microstructure was found to be increased using the UHRCT [4]. Greater anatomic detail is of great benefit to the surgeon intervening in and around this eloquent region. Hiraumi, et al., also showed that the detailed anatomic structures of the temporal bone were better seen on UHRCT.

UHRCT does have certain limitations. Bozzato, et al., investigated the accuracy of measurements of stapes prostheses with high resolution CT. The prostheses measurements were overestimated on the UHRCT [5]. Using high resolution FPCT, measurements of ossicular prostheses may become more accurate [5]. Due to the orientation of the stapes, accurate imaging on conventional HRCT is difficult. The stapes was found to be better evaluated in an oblique axial plane [6]. Manipulating the imaging data in a 3D plane would provide an advantageous look at not only the stapes, but also at its relationship with surrounding structures.
Superior semicircular canal dehiscence was also found to be overestimated on HRCT [7]. The amount of radio graphically diagnosed cases did not correlate with corresponding clinical symptoms [7]. The highly detailed evaluation of FPCT temporal bone images in a 3D workspace may bring to light more incidental findings and this could potentially be one negative aspect of this type of evaluation.

Patient radiation dose can be an issue with this type of high spatial resolution imaging. Studies have looked at ways of reducing the patient dose while maintaining the high resolution diagnostic quality including removing the anti-scatter grid [8]. There is much utility of FPCT in several other situations including identifying electrode contact position in cochlear implants in frequency evaluations [9]. A number of other pathologic conditions have been shown to be more conspicuous on FPCT in the literature including otosclerosis [10] and the possibilities for future utility of this type of imaging evaluation are several fold.

Although this is only a preliminary study demonstrating the potential utility of this type of imaging evaluation, there were some limitations. For example, the sample size was small. We intended to only demonstrate the potential capability of this type of imaging modality and further studies with larger sample sizes can be completed. In addition, only a few pathologic entities were evaluated in $3 \mathrm{D}$. In future studies, additional pathologic processes of the temporal bone can be further evaluated.

\section{Conclusion}

This preliminary report looked at the relationship of the normal and pathologic temporal bone using data from ultra-high resolution flat panel CT and virtually manipulating it in a three-dimensional workspace. Both the normal and pathologic temporal bone structures were seen clearly and their relationships to surrounding structures understood in greater detail in the 3D workspace. The ability to view and manipulate this high resolution imaging data in $3 \mathrm{D}$ would greatly benefit surgeons in pre and postoperative evaluation. Although the sample size in this preliminary report is small, the findings pave the way for future studies evaluating a larger sample size. Evaluation of normal anatomy and pathologic temporal bone entities can be viewed in obliquities and 3D orientations not able to be reproduced on conventional imaging techniques. The advantages of this level of detailed evaluation for the surgeon or clinician is not yet known, however this paper presents a pictorial review of high resolution FPCT data in a three-dimensional workspace.

\section{References}

1. Yamashita K, Hiwatashi A, Togao O, Kikuchi K, Matsumoto N, et al. (2018) Ultrahigh-resolution CT scan of the temporal bone. Eur Arch Otorhinolaryngol 275: 2797-2803.

2. Gupta R, Cheung AC, Bartling SH, Lisauskas J, Grasruck M, et al. (2008) Flat-panel volume CT: Fundamental principles, technology, and applications. Radiographics 28: 2009-2022.

3. Bartling SH, Shukla V, Becker H, Brady TJ, Hayman A, et al. (2005) High-resolution flat-panel volume-CT of temporal bone: Part 1: Axial preoperative anatomy. J comput Assist Tomogr 29: 420-423.

4. Hiraumi H, Obara M, Yoshioka K, Eharab S, Sato H (2019) Detectability of minute temporal bone structures with ultra-high resolution CT. Auris Nasis Larynx. Pg no: 830-835. 
5. Bozzato A, Struffert T, Hertel V, Iro H, Hornung (2010) Analysis of the accuracy of high-resolution computed tomography techniques for the measurements of stapes prostheses. Eur Radiol 20: 566-571.

6. Henrot P, lochum S, Batch T, Coffinet L, Blum A, et al. (2005) Current multiplanar imaging of the stapes. AJNR Am J Neuroradiol 26: 2128 2133.

7. Re M, Gioacchini FM, Salvolini U, Totaro AM, Santarelli A, et al. (2003) Multislice computed tomography overestimates superior semicircular canal dehiscence syndrome. Ann Otol Rhinol Laryngol 122: 625-631.
8. Mekabaty AE, Pross SE, Martinez M, Carey JP, Pearl MS (2018) Reducing radiation dose for high-resolution flat-panel CT imaging of superior semicircular canal dehiscence. Otol Neurotol 39: 683-690.

9. Jiam NT, Pearl MS, Carver C, Limb CJ (2016) Flat-panel CT imaging for individualized pitch mapping in cochlear implant users. Otol Nueorotol 37: $672-679$

10. Brown LA, Mocan BO, Redleaf MI (2019) Diagnostic protocol for detecting otosclerosis on high-resolution temporal bone CT. Ann Otol Rhinol Laryngol 9: 3489419859036. 


\section{di}

Journal of Anesthesia \& Clinical Care

Journal of Addiction \& Addictive Disorders

Advances in Microbiology Research

Advances in Industrial Biotechnology

Journal of Agronomy \& Agricultural Science

Journal of AIDS Clinical Research \& STDs

Journal of Alcoholism, Drug Abuse \& Substance Dependence

Journal of Allergy Disorders \& Therapy

Journal of Alternative, Complementary \& Integrative Medicine

Journal of Alzheimer's \& Neurodegenerative Diseases

Journal of Angiology \& Vascular Surgery

Journal of Animal Research \& Veterinary Science

Archives of Zoological Studies

Archives of Urology

Journal of Atmospheric \& Earth-Sciences

Journal of Aquaculture \& Fisheries

Journal of Biotech Research \& Biochemistry

Journal of Brain \& Neuroscience Research

Journal of Cancer Biology \& Treatment

Journal of Cardiology: Study \& Research

Journal of Cell Biology \& Cell Metabolism

Journal of Clinical Dermatology \& Therapy

Journal of Clinical Immunology \& Immunotherapy

Journal of Clinical Studies \& Medical Case Reports

Journal of Community Medicine \& Public Health Care

Current Trends: Medical \& Biological Engineering

Journal of Cytology \& Tissue Biology

Journal of Dentistry: Oral Health \& Cosmesis

Journal of Diabetes \& Metabolic Disorders

Journal of Dairy Research \& Technology

Journal of Emergency Medicine Trauma \& Surgical Care

Journal of Environmental Science: Current Research

Journal of Food Science \& Nutrition

Journal of Forensic, Legal \& Investigative Sciences

Journal of Gastroenterology \& Hepatology Research

Journal of Gerontology \& Geriatric Medicine
Journal of Genetics \& Genomic Sciences

Journal of Hematology, Blood Transfusion \& Disorders

Journal of Human Endocrinology

Journal of Hospice \& Palliative Medical Care

Journal of Internal Medicine \& Primary Healthcare

Journal of Infectious \& Non Infectious Diseases

Journal of Light \& Laser: Current Trends

Journal of Modern Chemical Sciences

Journal of Medicine: Study \& Research

Journal of Nanotechnology: Nanomedicine \& Nanobiotechnology

Journal of Neonatology \& Clinical Pediatrics

Journal of Nephrology \& Renal Therapy

Journal of Non Invasive Vascular Investigation

Journal of Nuclear Medicine, Radiology \& Radiation Therapy

Journal of Obesity \& Weight Loss

Journal of Orthopedic Research \& Physiotherapy

Journal of Otolaryngology, Head \& Neck Surgery

Journal of Protein Research \& Bioinformatics

Journal of Pathology Clinical \& Medical Research

Journal of Pharmacology, Pharmaceutics \& Pharmacovigilance

Journal of Physical Medicine, Rehabilitation \& Disabilities

Journal of Plant Science: Current Research

Journal of Psychiatry, Depression \& Anxiety

Journal of Pulmonary Medicine \& Respiratory Research

Journal of Practical \& Professional Nursing

Journal of Reproductive Medicine, Gynaecology \& Obstetrics

Journal of Stem Cells Research, Development \& Therapy

Journal of Surgery: Current Trends \& Innovations

Journal of Toxicology: Current Research

Journal of Translational Science and Research

Trends in Anatomy \& Physiology

Journal of Vaccines Research \& Vaccination

Journal of Virology \& Antivirals

Archives of Surgery and Surgical Education

Sports Medicine and Injury Care Journal

International Journal of Case Reports and Therapeutic Studies

Journal of Ecology Research and Conservation Biology 